\title{
CLOSED FORMS FOR DERANGEMENT NUMBERS IN TERMS OF THE HESSENBERG DETERMINANTS
}

\author{
FENG QI \\ Institute of Mathematics, Henan Polytechnic University, Jiaozuo 454010 , \\ Henan Province, China; College of Mathematics, Inner Mongolia University for \\ Nationalities, Tongliao 028043, Inner Mongolia Autonomous Region, China; \\ Department of Mathematics, College of Science, Tianjin Polytechnic University, \\ Tianjin 300387, China
}

\section{JIAO-LIAN ZHAO}

Department of Mathematics and Informatics, Weinan Teachers University, Weinan 714000, Shaanxi Province, China

\section{BAI-NI GUO}

School of Mathematics and Informatics, Henan Polytechnic University, Jiaozuo 454010, Henan Province, China

\begin{abstract}
In the paper, the authors find closed forms for derangement numbers in terms of the Hessenberg determinants, discover a recurrence relation of derangement numbers, present a formula for any higher order derivative of the exponential generating function of derangement numbers, and compute some related Hessenberg and tridiagonal determinants.
\end{abstract}

\section{MAIN RESUlts}

A square matrix $H=\left(h_{i j}\right)_{n \times n}$ is called a tridiagonal matrix if $h_{i j}=0$ for all pairs $(i, j)$ such that $|i-j|>1$. A tridiagonal determinant is a determinant with nonzero elements only on the diagonal and slots horizontally or vertically adjacent the diagonal. See the paper [6] and closely-related references therein.

E-mail addresses: qifeng618@gmail.com, qifeng618@hotmail.com, zhaoj12004@gmail.com, darren2004@126.com, bai.ni.guo@gmail.com, bai.ni.guo@hotmail.com

2010 Mathematics Subject Classification. 05A05; 05A10; 05A15; 11B37; 11B39; 11B83; 11B65; 11B83; 11C08; 11C20; 11Y55; 15A15; 65F40.

Key words and phrases. derangement number; closed form; Hessenberg determinant; tridiagonal determinant; generating function; recurrence relation; derivative. 
A matrix $H=\left(h_{i j}\right)_{n \times n}$ is called a lower (or an upper, respectively) Hessenberg matrix if $h_{i j}=0$ for all pairs $(i, j)$ such that $i+1<j$ (or $j+1<i$, respectively). See the paper [7] and closely-related references therein.

In mathematics, a closed expression is a mathematical form that can be evaluated in a finite number of operations. It may contain constants, variables, four arithmetic operations, and elementary functions, but usually no limit.

In combinatorial mathematics, a derangement is a permutation of the elements of a set, such that no element appears in its original position. The number of derangements of a set of size $n$ is called the derangement number and usually denoted by $! n$. The problem of counting derangements was first considered in 1708 and solved in 1713 both by Pierre Raymond de Montmort, as did Nicholas Bernoulli at about the same time. The first eleven derangement numbers ! $n$ for $0 \leq n \leq 10$ are $1,0,1,2,9,44,265,1854,14833,133496,1334961$.

Derangement numbers $! n$ can be generated by the exponential generating function

$$
D(x)=\frac{e^{-x}}{1-x}=\frac{1}{e^{x}(1-x)}=\sum_{n=0}^{\infty} ! n \frac{x^{n}}{n !} .
$$

For more and detailed information on derangement numbers ! $n$, please refer to 1 , 2, 17, 18, and plenty of references therein.

In the papers [8, 14, 15], the authors recovered that derangement numbers !n can be represented by a tridiagonal $(n+1) \times(n+1)$ determinant

$$
! n=-\left|\begin{array}{ccccccccc}
-1 & 1 & 0 & 0 & 0 & \ldots & 0 & 0 & 0 \\
0 & 0 & 1 & 0 & 0 & \ldots & 0 & 0 & 0 \\
0 & -1 & 1 & 1 & 0 & \ldots & 0 & 0 & 0 \\
0 & 0 & -2 & 2 & 1 & \ldots & 0 & 0 & 0 \\
0 & 0 & 0 & -3 & 3 & \ldots & 0 & 0 & 0 \\
\vdots & \vdots & \vdots & \vdots & \vdots & \ddots & \vdots & \vdots & \vdots \\
0 & 0 & 0 & 0 & 0 & \ldots & n-3 & 1 & 0 \\
0 & 0 & 0 & 0 & 0 & \ldots & -(n-2) & n-2 & 1 \\
0 & 0 & 0 & 0 & 0 & \ldots & 0 & -(n-1) & n-1
\end{array}\right|
$$

for $n \in\{0\} \cup \mathbb{N}$.

In this paper, by considering the generating function $\frac{1}{e^{x}(1-x)}$ in (1), we represent derangement numbers $! n$ in terms of the Hessenberg determinants as follows.

Theorem 1. For $n \geq 0$, derangement numbers $! n$ can be computed by

$$
\begin{aligned}
! n & =\left|\begin{array}{ccccccc}
1 & -1 & 0 & \ldots & 0 & 0 & 0 \\
0 & 0 & -1 & \cdots & 0 & 0 & 0 \\
0 & \left(\begin{array}{l}
2 \\
0
\end{array}\right) & 0 & \ldots & 0 & 0 & 0 \\
0 & \left(\begin{array}{c}
3 \\
0
\end{array}\right) 2 & \left(\begin{array}{c}
3 \\
1
\end{array}\right) & \cdots & 0 & 0 & 0 \\
\vdots & \vdots & \vdots & \ddots & \vdots & \vdots & \vdots \\
0 & \left(\begin{array}{c}
n-3 \\
0
\end{array}\right)(n-4) & \left(\begin{array}{c}
n-3 \\
1
\end{array}\right)(n-5) & \ldots & -1 & 0 & 0 \\
0 & \left(\begin{array}{c}
n-2 \\
0
\end{array}\right)(n-3) & \left(\begin{array}{c}
n-2 \\
1
\end{array}\right)(n-4) & \ldots & 0 & -1 & 0 \\
0 & \left(\begin{array}{c}
n-1 \\
0
\end{array}\right)(n-2) & \left(\begin{array}{c}
n-1 \\
1
\end{array}\right)(n-3) & \ldots & \left(\begin{array}{c}
n-1 \\
n-3
\end{array}\right) & 0 & -1 \\
0 & \left(\begin{array}{c}
n \\
0
\end{array}\right)(n-1) & \left(\begin{array}{c}
n \\
1
\end{array}\right)(n-2) & \ldots & \left(\begin{array}{c}
n \\
n-3
\end{array}\right) 2 & \left(\begin{array}{c}
n \\
n-2
\end{array}\right) & 0
\end{array}\right|
\end{aligned}
$$


where $e_{1,1}=1, e_{i, 1}=0$ for $2 \leq i \leq n+1$, and

$$
e_{i j}= \begin{cases}\left(\begin{array}{l}
i-1 \\
j-2
\end{array}\right)(i-j), & i-j \geq 1 \\
0, & i-j<1\end{cases}
$$

for $1 \leq n+1$ and $2 \leq j \leq n+1$. Consequently,

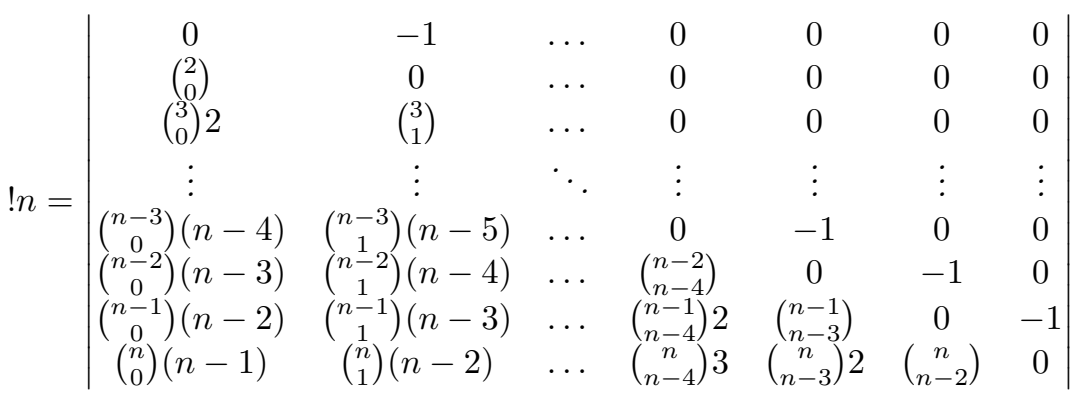

$$
\begin{aligned}
& =\left|q_{i j}\right|_{n \times n}
\end{aligned}
$$

for $n \in \mathbb{N}$, where

$$
q_{i j}= \begin{cases}\left(\begin{array}{c}
i \\
j-1
\end{array}\right)(i-j), & i-j \geq 1 \\
0, & i-j<1\end{cases}
$$

for $1 \leq n$ and $2 \leq j \leq n$.

As consequences of Theorem 1 and the equation (1), the following recurrence relations can be discovered readily.

Theorem 2. Derangement numbers !n meet

$$
! n=\sum_{i=0}^{n-2}\left(\begin{array}{c}
n \\
i
\end{array}\right)(n-i-1)(! i), \quad n \geq 2
$$

and

$$
n !=\sum_{k=0}^{n}\left(\begin{array}{l}
n \\
k
\end{array}\right)(! k)=\sum_{k=0}^{n}\left(\begin{array}{l}
n \\
k
\end{array}\right)[!(n-k)], \quad n \geq 0 .
$$

By induction, we also present an explicit formula for the $n$th derivative of the exponential generating function $D(x)$ as follows.

Theorem 3. For $n \in\{0\} \cup \mathbb{N}$, the nth derivative of the generating function $D(x)$ can be computed by

$$
\frac{\mathrm{d}^{n}}{\mathrm{~d} x^{n}}\left(\frac{e^{-x}}{1-x}\right)=\frac{e^{-x}}{(1-x)^{n+1}} \sum_{i=0}^{n} a_{n, i} x^{i}
$$

where

$$
a_{n, i}=\frac{\langle n\rangle_{i}[!(n-i)]}{i !}
$$

and

$$
\langle x\rangle_{n}=\prod_{k=0}^{n-1}(x-k)= \begin{cases}x(x-1) \cdots(x-n+1), & n \geq 1 \\ 1, & n=0\end{cases}
$$

stands for the falling factorial. 
As a consequence of Theorem 3, a formula for some Hessenberg and tridiagonal determinants are established as follows.

Corollary 1. For $n \in \mathbb{N}$, let

$$
e_{i j}(x)= \begin{cases}\left(\begin{array}{c}
i \\
j-1
\end{array}\right)(i-j+x), & i-j+1 \geq 0 \\
0, & i-j+1<0\end{cases}
$$

and

$$
h_{i j}(x)= \begin{cases}1-x, & i-j=-1, \\ 1-i-x, & i-j=0, \\ 1-i, & i-j=1, \\ 0, & i-j \neq 0, \pm 1\end{cases}
$$

for all $1 \leq i, j \leq n$. Then the Hessenberg and tridiagonal determinants $\left|e_{i j}(x)\right|_{n \times n}$ and $\left|h_{i j}(x)\right|_{n \times n}$ can be computed by

$$
\left|e_{i j}(x)\right|_{n \times n}=(-1)^{n}\left|h_{i j}(x)\right|_{n \times n}=\sum_{i=0}^{n}\langle n\rangle_{i}[!(n-i)] \frac{x^{i}}{i !},
$$

where $\langle n\rangle_{i}$ is defined by 9 .

\section{A LEMMA}

For supplying a concise proof for Theorem 1 , we need the following lemma which was concluded in [9, Section 2.2, p. 849], [10, p. 94], [13, Remark 6], and [16, Lemma 2.1] from [3, p. 40, Exercise 5)].

Lemma 1. Let $u(x)$ and $v(x) \neq 0$ be differentiable functions, let $U_{(n+1) \times 1}(x)$ be an $(n+1) \times 1$ matrix whose elements $u_{k, 1}(x)=u^{(k-1)}(x)$ for $1 \leq k \leq n+1$, let $V_{(n+1) \times n}(x)$ be an $(n+1) \times n$ matrix whose elements

$$
v_{i, j}(x)= \begin{cases}\left(\begin{array}{l}
i-1 \\
j-1
\end{array}\right) v^{(i-j)}(x), & i-j \geq 0 \\
0, & i-j<0\end{cases}
$$

for $1 \leq i \leq n+1$ and $1 \leq j \leq n$, and let $\left|W_{(n+1) \times(n+1)}(x)\right|$ denote the lower Hessenberg determinant of the $(n+1) \times(n+1)$ lower Hessenberg matrix

$$
W_{(n+1) \times(n+1)}(x)=\left[U_{(n+1) \times 1}(x) \quad V_{(n+1) \times n}(x)\right] .
$$

Then the nth derivative of the ratio $\frac{u(x)}{v(x)}$ can be computed by

$$
\frac{\mathrm{d}^{n}}{\mathrm{~d} x^{n}}\left[\frac{u(x)}{v(x)}\right]=(-1)^{n} \frac{\left|W_{(n+1) \times(n+1)}(x)\right|}{v^{n+1}(x)} .
$$

We remark that Lemma 1 is an effectual tool to express some mathematical quantities such as the Bernoulli numbers and polynomials, the Euler numbers and polynomials, and the Fibonacci numbers and polynomials as the Hessenberg or tridiagonal determinants. For more information, please refer to [9, 10, 12, 16] and closely-related references therein. 


\section{Proofs of Theorems 1 to 3 and Corollary 1}

Now we are in a position to provide proofs for Theorems 1 to 3 and Corollary 1 respectively.

Proof of Theorem 1. Let $v(x)=e^{x}(1-x)$. It is not difficult to verify by induction that

$$
v^{(k)}(x)=-e^{x}(k-1+x) \rightarrow 1-k
$$

as $x \rightarrow 0$ for $k \geq 0$.

Applying $u(x)=1$ and $v(x)=e^{x}(1-x)$ in Lemma 1 yields that $u_{1,1}(x)=1$ and $u_{k, 1}(x)=0$ for $2 \leq k \leq n+1$, while

$$
\begin{aligned}
v_{i, j}(x) & = \begin{cases}\left(\begin{array}{l}
i-1 \\
j-1
\end{array}\right) v^{(i-j)}(x), & i-j \geq 0 \\
0, & i-j<0\end{cases} \\
& = \begin{cases}-\left(\begin{array}{l}
i-1 \\
j-1
\end{array}\right) e^{x}(i-j-1+x), & i-j \geq 0 \\
0, & i-j<0\end{cases} \\
& \rightarrow \begin{cases}-\left(\begin{array}{l}
i-1 \\
j-1
\end{array}\right)(i-j-1), & i-j \geq 0 \\
0, & i-j<0\end{cases}
\end{aligned}
$$

as $x \rightarrow 0$ for $1 \leq i \leq n+1$ and $1 \leq j \leq n$. Consequently, by virtue of the formula (11), we have

$$
\begin{aligned}
& \frac{\mathrm{d}^{n} D(x)}{\mathrm{d} x^{n}}=\frac{(-1)^{n}}{\left[e^{x}(1-x)\right]^{n+1}} \mid \begin{array}{ccc}
1 & -e^{x}(-1+x) & 0 \\
0 & -\left(\begin{array}{c}
1 \\
0
\end{array}\right) e^{x} x & -e^{x}(-1+x) \\
0 & -\left(\begin{array}{l}
2 \\
0
\end{array}\right) e^{x}(1+x) & -\left(\begin{array}{c}
2 \\
1
\end{array}\right) e^{x} x \\
0 & -\left(\begin{array}{c}
3 \\
0
\end{array}\right) e^{x}(2+x) & -\left(\begin{array}{c}
3 \\
1
\end{array}\right) e^{x}(1+x) \\
\vdots & \vdots & \vdots \\
0 & -\left(\begin{array}{c}
n-3 \\
0
\end{array}\right) e^{x}(n-4+x) & -\left(\begin{array}{c}
n-3 \\
1
\end{array}\right) e^{x}(n-5+x) \\
0 & -\left(\begin{array}{c}
n-2 \\
0
\end{array}\right) e^{x}(n-3+x) & -\left(\begin{array}{c}
n-2 \\
0
\end{array}\right) e^{x}(n-4+x) \\
0 & -\left(\begin{array}{c}
n-1 \\
0
\end{array}\right) e^{x}(n-2+x) & -\left(\begin{array}{c}
n-1 \\
1
\end{array}\right) e^{x}(n-3+x) \\
0 & -\left(\begin{array}{c}
n \\
0
\end{array}\right) e^{x}(n-1+x) & -\left(\begin{array}{c}
n \\
1
\end{array}\right) e^{x}(n-2+x)
\end{array}
\end{aligned}
$$

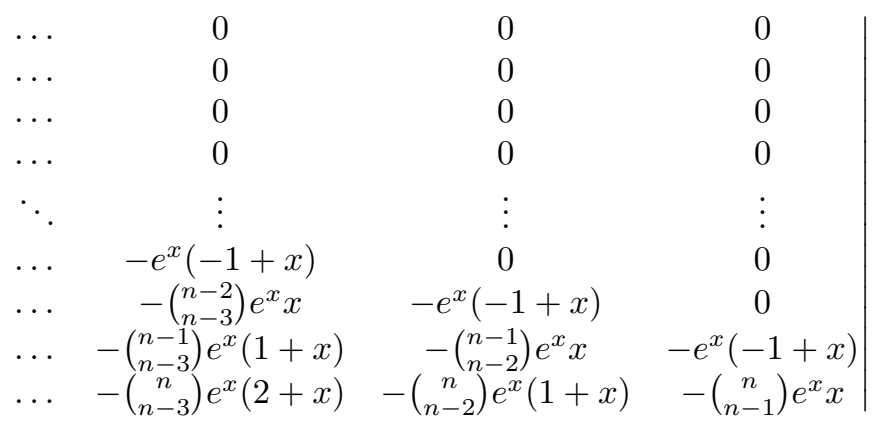




$$
\begin{aligned}
& \rightarrow(-1)^{n}\left|\begin{array}{cccccc}
1 & -(-1) & 0 & \cdots & 0 & 0 \\
0 & 0 & -(-1) & \cdots & 0 & 0 \\
0 & -\left(\begin{array}{c}
2 \\
0
\end{array}\right) & 0 & \cdots & 0 & 0 \\
0 & -\left(\begin{array}{l}
3 \\
0
\end{array}\right) 2 & -\left(\begin{array}{l}
3 \\
1
\end{array}\right) & \cdots & 0 & 0 \\
\vdots & \vdots & \vdots & \ddots & \vdots & \vdots \\
0 & -\left(\begin{array}{c}
n-3 \\
0
\end{array}\right)(n-4) & -\left(\begin{array}{c}
n-3 \\
1
\end{array}\right)(n-5) & \ldots & 0 & 0 \\
0 & -\left(\begin{array}{c}
n-2 \\
0
\end{array}\right)(n-3) & -\left(\begin{array}{c}
n-2 \\
n
\end{array}\right)(n-4) & \ldots & -(-1) & 0 \\
0 & -\left(\begin{array}{c}
n-1 \\
0
\end{array}\right)(n-2) & -\left(\begin{array}{c}
n-1 \\
1
\end{array}\right)(n-3) & \ldots & 0 & -(-1) \\
0 & -\left(\begin{array}{c}
n \\
0
\end{array}\right)(n-1) & -\left(\begin{array}{c}
n \\
n \\
1
\end{array}\right)(n-2) & \ldots & -\left(\begin{array}{c}
n \\
n-2
\end{array}\right) & 0
\end{array}\right| \\
& =\left|\begin{array}{ccccccc}
1 & -1 & 0 & \ldots & 0 & 0 & 0 \\
0 & 0 & -1 & \ldots & 0 & 0 & 0 \\
0 & \left(\begin{array}{l}
2 \\
0
\end{array}\right) & 0 & \ldots & 0 & 0 & 0 \\
0 & \left(\begin{array}{c}
3 \\
0
\end{array}\right) 2 & \left(\begin{array}{c}
3 \\
1
\end{array}\right) & \ldots & 0 & 0 & 0 \\
\vdots & \vdots & \vdots & \ddots & \vdots & \vdots & \vdots \\
0 & \left(\begin{array}{c}
n-3 \\
0
\end{array}\right)(n-4) & \left(\begin{array}{c}
n-3 \\
1
\end{array}\right)(n-5) & \ldots & -1 & 0 & 0 \\
0 & \left(\begin{array}{c}
n-2 \\
0
\end{array}\right)(n-3) & \left(\begin{array}{c}
n-2 \\
n
\end{array}\right)(n-4) & \ldots & 0 & -1 & 0 \\
0 & \left(\begin{array}{c}
n-1 \\
0
\end{array}\right)(n-2) & \left(\begin{array}{c}
n-1 \\
1
\end{array}\right)(n-3) & \ldots & \left(\begin{array}{c}
n-1 \\
n-3
\end{array}\right) & 0 & -1 \\
0 & \left(\begin{array}{c}
n \\
n \\
0
\end{array}\right)(n-1) & \left(\begin{array}{c}
n \\
n \\
1
\end{array}\right)(n-2) & \ldots & \left(\begin{array}{c}
n \\
n-3
\end{array}\right) 2 & 0
\end{array}\right|
\end{aligned}
$$

as $x \rightarrow 0$ for $n \geq 0$. Therefore, since $D(x)$ is a generating function of $! n$, as showed in (1), we obtain

$$
! n=\lim _{x \rightarrow 0} \frac{\mathrm{d}^{n} D(x)}{\mathrm{d} x^{n}}=\left|\begin{array}{cccccc}
1 & -1 & 0 & \cdots & 0 & 0 \\
0 & 0 & -1 & \cdots & 0 & 0 \\
0 & \left(\begin{array}{c}
2 \\
0
\end{array}\right) & 0 & \cdots & 0 & 0 \\
0 & \left(\begin{array}{l}
3 \\
0
\end{array}\right) 2 & \left(\begin{array}{c}
3 \\
1
\end{array}\right) & \cdots & 0 & 0 \\
\vdots & \vdots & \vdots & \ddots & \vdots & \vdots \\
0 & \left(\begin{array}{c}
n-3 \\
0
\end{array}\right)(n-4) & \left(\begin{array}{c}
n-3 \\
1
\end{array}\right)(n-5) & \ldots & 0 & 0 \\
0 & \left(\begin{array}{c}
n-2 \\
0
\end{array}\right)(n-3) & \left(\begin{array}{c}
n-2 \\
n
\end{array}\right)(n-4) & \ldots & -1 & 0 \\
0 & \left(\begin{array}{c}
n-1 \\
0
\end{array}\right)(n-2) & \left(\begin{array}{c}
n-1 \\
1
\end{array}\right)(n-3) & \ldots & 0 & -1 \\
0 & \left(\begin{array}{c}
n \\
0
\end{array}\right)(n-1) & \left(\begin{array}{c}
n \\
n \\
1
\end{array}\right)(n-2) & \ldots & \left(\begin{array}{c}
n \\
n-2
\end{array}\right) & 0
\end{array}\right| .
$$

The proof of Theorem 1 is complete.

Proof of Theorem 2. The recurrence relation (5) immediately follows from expanding the determinant (3) according to the last columns consecutively.

The equation (1) can also be rewritten as

$$
\begin{gathered}
\frac{1}{1-x}=e^{x} \sum_{n=0}^{\infty} ! n \frac{x^{n}}{n !}, \quad \sum_{n=0}^{\infty} x^{n}=\sum_{n=0}^{\infty} \frac{x^{n}}{n !} \sum_{n=0}^{\infty} ! n \frac{x^{n}}{n !}, \\
\sum_{n=0}^{\infty} x^{n}=\sum_{n=0}^{\infty}\left[\sum_{k=0}^{n} \frac{1}{k !} \frac{(n-k)}{(n-k) !}\right] x^{n}=\sum_{n=0}^{\infty}\left[\sum_{k=0}^{n} \frac{! k}{k !} \frac{1}{(n-k) !}\right] x^{n} .
\end{gathered}
$$

As a result, by equating the last equality and rearranging, we obtain the identity (6). The proof of Theorem 2 is complete.

Proof of Theorem 3. By the equation (1), it is not difficult to see that the equality $a_{n, 0}=! n$ holds for all $n \geq 0$. 
A direct computation gives

$$
\frac{\mathrm{d}}{\mathrm{d} x}\left(\frac{e^{-x}}{1-x}\right)=\frac{e^{-x}}{(1-x)^{2}} x \quad \text { and } \quad \frac{\mathrm{d}^{2}}{\mathrm{~d} x^{2}}\left(\frac{e^{-x}}{1-x}\right)=\frac{e^{-x}}{(1-x)^{3}}\left(1+x^{2}\right) .
$$

It is clear that, when $n=0,1,2$, the equality (7) is valid respectively.

Assume that the equality (7) is valid for some $n \geq 3$. By this inductive hypothesis, we have

$$
\begin{aligned}
& \frac{\mathrm{d}^{n+1}}{\mathrm{~d} x^{n+1}}\left(\frac{e^{-x}}{1-x}\right)=\frac{\mathrm{d}}{\mathrm{d} x}\left[\frac{\mathrm{d}^{n}}{\mathrm{~d} x^{n}}\left(\frac{e^{-x}}{1-x}\right)\right]=\frac{\mathrm{d}}{\mathrm{d} x}\left[\frac{e^{-x}}{(1-x)^{n+1}} \sum_{i=0}^{n} a_{n, i} x^{i}\right] \\
= & \frac{e^{-x}}{(1-x)^{n+2}}\left[(n+x) \sum_{i=0}^{n} a_{n, i} x^{i}-(x-1) \sum_{i=1}^{n} a_{n, i} i x^{i-1}\right] \\
= & \frac{e^{-x}}{(1-x)^{n+2}}\left[n \sum_{i=0}^{n} a_{n, i} x^{i}+\sum_{i=0}^{n} a_{n, i} x^{i+1}-\sum_{i=1}^{n} a_{n, i} i x^{i}+\sum_{i=1}^{n} a_{n, i} i x^{i-1}\right] \\
= & \frac{e^{-x}}{(1-x)^{n+2}}\left[n \sum_{i=0}^{n} a_{n, i} x^{i}+\sum_{i=1}^{n+1} a_{n, i-1} x^{i}-\sum_{i=1}^{n} a_{n, i} i x^{i}+\sum_{i=0}^{n-1} a_{n, i+1}(i+1) x^{i}\right] \\
= & \frac{e^{-x}}{(1-x)^{n+2}}\left[n a_{n, 0}+n \sum_{i=1}^{n-1} a_{n, i} x^{i}+n a_{n, n} x^{n}+\sum_{i=1}^{n-1} a_{n, i-1} x^{i}+a_{n, n-1} x^{n}\right. \\
& \left.+a_{n, n} x^{n+1}-\sum_{i=1}^{n-1} a_{n, i} i x^{i}-a_{n, n} n x^{n}+a_{n, 1}+\sum_{i=1}^{n-1} a_{n, i+1}(i+1) x^{i}\right] \\
= & \frac{e^{-x}}{(1-x)^{n+2}}\left[n a_{n, 0}+a_{n, 1}+\sum_{i=1}^{n-1}\left[a_{n, i-1}+(n-i) a_{n, i}+(i+1) a_{n, i+1}\right] x^{i}\right. \\
& \left.+a_{n, n-1} x^{n}+a_{n, n} x^{n+1}\right]
\end{aligned}
$$

and

$$
\frac{\mathrm{d}^{n+1}}{\mathrm{~d} x^{n+1}}\left(\frac{e^{-x}}{1-x}\right)=\frac{e^{-x}}{(1-x)^{n+2}} \sum_{i=0}^{n+1} a_{n+1, i} x^{i} .
$$

Equating the above two equalities yields

$$
\begin{aligned}
a_{n+1,0} & =n a_{n, 0}+a_{n, 1}, \\
a_{n+1, n} & =a_{n, n-1}, \\
a_{n+1, n+1} & =a_{n, n},
\end{aligned}
$$

and

$$
a_{n+1, i}=a_{n, i-1}+(n-i) a_{n, i}+(i+1) a_{n, i+1}, \quad 1 \leq i \leq n-1 .
$$

Since $a_{1,0}=0$ and $a_{0,0}=a_{1,1}=1$, the recurrence relations (13) and 14 implies $a_{n, n-1}=0$ and $a_{n, n}=1$.

From (12) and $a_{n, 0}=! n$, it follows that

$$
a_{n, 1}=a_{n+1,0}-n a_{n, 0}=!(n+1)-n(! n)=n[!(n-1)],
$$

where the well-known recurrence relation

$$
! n=(n-1)[!(n-1)+!(n-2)], \quad n \geq 2
$$


was employed.

By virtue of the recurrence relations 15 and 16 and the identities $a_{n, 0}=! n$ and $a_{n, 1}=n[!(n-1)]$, we obtain

$$
\begin{gathered}
a_{n, 2}=\frac{a_{n+1,1}-a_{n, 0}-(n-1) a_{n, 1}}{2}=\frac{(n+1)(! n)-! n-(n-1) n[!(n-1)]}{2} \\
=\frac{n(! n)-(n-1) n[!(n-1)]}{2} \\
=\frac{n\{! n-(n-1)[!(n-1)]\}}{2}=\frac{n(n-1)[!(n-2)]}{2} .
\end{gathered}
$$

Similarly, we have

$$
\begin{gathered}
a_{n, 3}=\frac{a_{n+1,2}-a_{n, 1}-(n-2) a_{n, 2}}{3} \\
=\frac{1}{3}\left\{\frac{(n+1) n[!(n-1)]}{2}-n[!(n-1)]-(n-2) \frac{n(n-1)[!(n-2)]}{2}\right\} \\
=\frac{1}{3}\left\{\frac{(n-1) n[!(n-1)]}{2}-(n-2) \frac{n(n-1)[!(n-2)]}{2}\right\} \\
=\frac{(n-1) n}{6}\{[!(n-1)]-(n-2)[!(n-2)]\}=\frac{n(n-1)(n-2)[!(n-3)]}{6}
\end{gathered}
$$

and

$$
\begin{gathered}
a_{n, 4}=\frac{a_{n+1,3}-a_{n, 2}-(n-3) a_{n, 3}}{4}=\frac{1}{4}\left\{\frac{(n+1) n(n-1)[!(n-2)]}{6}\right. \\
\left.-\frac{n(n-1)[!(n-2)]}{2}-(n-3) \frac{n(n-1)(n-2)[!(n-3)]}{6}\right\} \\
=\frac{n(n-1)(n-2)}{24}\{[!(n-2)]-(n-3)[!(n-3)]\} \\
=\frac{n(n-1)(n-2)(n-3)[!(n-4)]}{24} .
\end{gathered}
$$

Inductively, we conclude the relation (8). The proof of Theorem 3 is complete.

Proof of Corollary 1. From the proof of Theorem 1, it follows that

$$
\begin{aligned}
& \frac{\mathrm{d}^{n}}{\mathrm{~d} x^{n}}\left(\frac{e^{-x}}{1-x}\right)=\frac{\mathrm{d}^{n}}{\mathrm{~d} x^{n}}\left[\frac{1}{e^{x}(1-x)}\right]=\frac{1}{(1-x)^{n+1} e^{x}}
\end{aligned}
$$

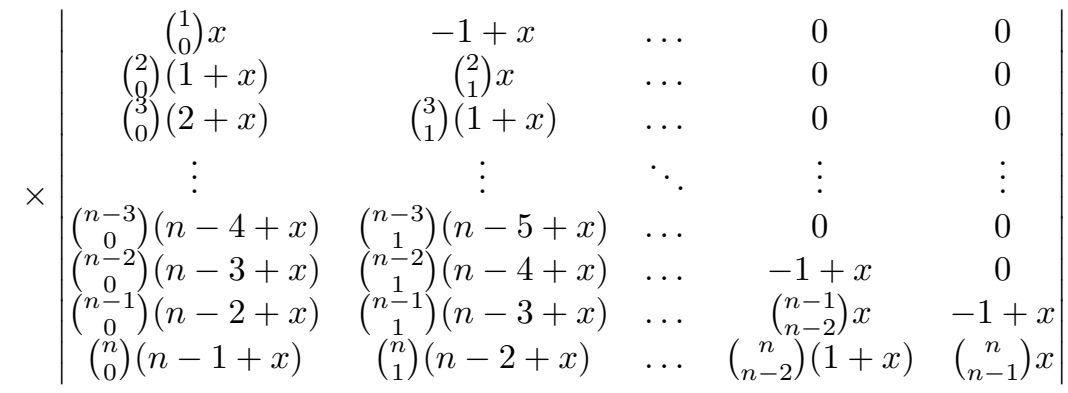

$$
\begin{aligned}
& =\frac{e^{-x}}{(1-x)^{n+1}}\left|e_{i j}(x)\right|_{n \times n}
\end{aligned}
$$

for $n \in \mathbb{N}$. Combining this with Theorem 3 leads to the equality constituted by the very ends of 10 . 
Applying $u(x)=e^{-x}$ and $v(x)=1-x$ in Lemma 1 gives

$$
u_{k, 1}=\left(e^{-x}\right)^{(k-1)}=(-1)^{k-1} e^{-x} \rightarrow(-1)^{k-1}
$$

for $1 \leq k \leq n+1$ as $x \rightarrow 0$ and

$$
\begin{gathered}
v_{i, j}=\left(\begin{array}{l}
i-1 \\
j-1
\end{array}\right)(1-x)^{(i-j)}= \begin{cases}\left(\begin{array}{l}
i-1 \\
j-1
\end{array}\right)(1-x), & i-j=0 \\
-\left(\begin{array}{l}
i-1 \\
j-1
\end{array}\right), & i-j=1 \\
0, & i-j \neq 0,1\end{cases} \\
=\left\{\begin{array} { l l } 
{ 1 - x , } & { i - j = 0 } \\
{ 1 - i , } & { i - j = 1 } \\
{ 0 , } & { i - j \neq 0 , 1 }
\end{array} \rightarrow \left\{\begin{array}{ll}
1, & i-j=0 \\
1-i, & i-j=1 \\
0, & i-j \neq 0,1
\end{array}\right.\right.
\end{gathered}
$$

for $1 \leq i \leq n+1$ and $1 \leq j \leq n$ as $x \rightarrow 0$. Consequently, by virtue of the formula $\sqrt{11}$, we have

$$
\begin{aligned}
& \frac{\mathrm{d}^{n} D(x)}{\mathrm{d} x^{n}}=\frac{(-1)^{n}}{(1-x)^{n+1}}\left|\begin{array}{cccccc}
e^{-x} & 1-x & 0 & \cdots & 0 & 0 \\
-e^{-x} & -1 & 1-x & \cdots & 0 & 0 \\
e^{-x} & 0 & -2 & \cdots & 0 & 0 \\
\vdots & \vdots & \vdots & \ddots & \vdots & \vdots \\
(-1)^{n-2} e^{-x} & 0 & 0 & \ldots & 1-x & 0 \\
(-1)^{n-1} e^{-x} & 0 & 0 & \ldots & -(n-1) & 1-x \\
(-1)^{n} e^{-x} & 0 & 0 & \ldots & 0 & -n
\end{array}\right| \\
& =\frac{(-1)^{n} e^{-x}}{(1-x)^{n+1}}\left|\begin{array}{cccccc}
-x & 1-x & 0 & \ldots & 0 & 0 \\
-1 & -1-x & 1-x & \ldots & 0 & 0 \\
0 & -2 & -2-x & \ldots & 0 & 0 \\
\vdots & \vdots & \vdots & \ddots & \vdots & \vdots \\
0 & 0 & 0 & \ldots & 1-x & 0 \\
0 & 0 & 0 & \ldots & 2-n-x & 1-x \\
0 & 0 & 0 & \ldots & 1-n & 1-n-x
\end{array}\right| \\
& =\frac{e^{-x}}{(1-x)^{n+1}}(-1)^{n}\left|h_{i j}(x)\right|_{n \times n},
\end{aligned}
$$

where $n \in \mathbb{N}$. Combining this with Theorem 3 results in the equality constituted by the right-hand one in 10 . The proof of Corollary 1 is complete.

\section{REMARKS}

Remark 1. The equation (1) can be rearranged as

$$
\begin{gathered}
e^{-x}=(1-x) \sum_{n=0}^{\infty} ! n \frac{x^{n}}{n !} \\
\sum_{n=0}^{\infty} \frac{(-1)^{n}}{n !} x^{n}=\sum_{n=0}^{\infty} ! n \frac{x^{n}}{n !}-\sum_{n=1}^{\infty} !(n-1) \frac{x^{n}}{(n-1) !} \\
\sum_{n=0}^{\infty} \frac{(-1)^{n}}{n !} x^{n}=1+\sum_{n=1}^{\infty}[! n-n \times !(n-1)] \frac{x^{n}}{n !}
\end{gathered}
$$


Hence, we recover the relation

$$
! n-n \times !(n-1)=(-1)^{n}, \quad n \in \mathbb{N} .
$$

Remark 2. The recurrence relation (5) can also be deduced from the expression (4).

Remark 3. Let $M_{0}=1$ and

$$
M_{n}=\left|\begin{array}{cccccc}
m_{1,1} & m_{1,2} & 0 & \ldots & 0 & 0 \\
m_{2,1} & m_{2,2} & m_{2,3} & \ldots & 0 & 0 \\
m_{3,1} & m_{3,2} & m_{3,3} & \ldots & 0 & 0 \\
\vdots & \vdots & \vdots & \vdots & \vdots & \vdots \\
m_{n-2,1} & m_{n-2,2} & m_{n-2,3} & \ldots & m_{n-2, n-1} & 0 \\
m_{n-1,1} & m_{n-1,2} & m_{n-1,3} & \ldots & m_{n-1, n-1} & m_{n-1, n} \\
m_{n, 1} & m_{n, 2} & m_{n, 3} & \ldots & m_{n, n-1} & m_{n, n}
\end{array}\right|
$$

for $n \in \mathbb{N}$. It was obtained in [4, p. 222, Theorem] that the sequence $M_{n}$ for $n \geq 0$ satisfies $M_{1}=m_{1,1}$ and

$$
M_{n}=m_{n, n} M_{n-1}+\sum_{r=1}^{n-1}\left[(-1)^{n-r} m_{n, r} \prod_{j=r}^{n-1} m_{j, j+1} M_{r-1}\right], \quad n \geq 2 .
$$

In particular, it was showed in [4, pp. 222-223, Examples 1 and 2] that

$$
\begin{aligned}
& \left|\begin{array}{cccccc}
1 & -1 & 0 & \cdots & 0 & 0 \\
1 & 2 & -1 & \cdots & 0 & 0 \\
1 & 1 & 2 & \cdots & 0 & 0 \\
\vdots & \vdots & \vdots & \vdots & \vdots & \vdots \\
1 & 1 & 1 & \cdots & -1 & 0 \\
1 & 1 & 1 & \cdots & 2 & -1 \\
1 & 1 & 1 & \cdots & 1 & 2
\end{array}\right|_{n \times n}=F_{2 n} \\
& \left|\begin{array}{cccccc}
2 & -1 & 0 & \ldots & 0 & 0 \\
1 & 2 & -1 & \ldots & 0 & 0 \\
1 & 1 & 2 & \cdots & 0 & 0 \\
\vdots & \vdots & \vdots & \vdots & \vdots & \vdots \\
1 & 1 & 1 & \cdots & -1 & 0 \\
1 & 1 & 1 & \cdots & 2 & -1 \\
1 & 1 & 1 & \cdots & 1 & 2
\end{array}\right|_{n \times n}=F_{2 n+1},
\end{aligned}
$$

and

where

$$
\left|\begin{array}{cccccc}
2 & 1 & 0 & \ldots & 0 & 0 \\
1 & 2 & 1 & \ldots & 0 & 0 \\
1 & 1 & 2 & \ldots & 0 & 0 \\
\vdots & \vdots & \vdots & \vdots & \vdots & \vdots \\
1 & 1 & 1 & \ldots & 1 & 0 \\
1 & 1 & 1 & \ldots & 2 & 1 \\
1 & 1 & 1 & \ldots & 1 & 2
\end{array}\right|_{n \times n}=F_{n+2}
$$

$$
F_{n}=\frac{(1+\sqrt{5})^{n}-(1-\sqrt{5})^{n}}{2^{n} \sqrt{5}}
$$


for $n \in \mathbb{N}$ denotes the Fibonacci number. For more information on the Fibonacci numbers $F_{n}$, please refer to [4, 5, 12] and closely-related references therein.

Applying (17) to (4) yields the recurrence relation (5) once again.

Remark 4. This paper is a companion of the articles or notes [8, 11, 14, 15].

\section{REFERENCES}

[1] M. Aigner, A Course in Enumeration, Graduate Texts in Mathematics, 238, Springer, Berlin, 2007.

[2] T. Andreescu and Z. Feng, A Path to Combinatorics for Undergraduates-Counting Strtegies, Birkhäuser, Boston-Basel-Berlin, 2004.

[3] N. Bourbaki, Elements of Mathematics: Functions of a Real Variable: Elementary Theory, Translated from the 1976 French original by Philip Spain. Elements of Mathematics (Berlin). Springer-Verlag, Berlin, 2004; Available online at http://dx.doi.org/10.1007/ 978-3-642-59315-4

[4] N. D. Cahill, J. R. D'Errico, D. A. Narayan, and J. Y. Narayan, Fibonacci determinants, College Math. J. 3 (2002), 221-225; Available online at http://dx.doi.org/10.2307/1559033

[5] C.-P. Chen, A.-Q. Liu, and F. Qi, Proofs for the limit of ratios of consecutive terms in Fibonacci sequence, Cubo Mat. Educ. 5 (2003), no. 3, 23-30.

[6] V. Higgins and C. Johnson, Inverse spectral problems for collections of leading principal submatrices of tridiagonal matrices, Linear Algebra Appl. 489 (2016), 104-122; Available online at http://dx.doi.org/10.1016/j.laa.2015.10.004

[7] R. S. Martin and J. H. Wilkinson, Handbook Series Linear Algebra: Similarity reduction of a general matrix to Hessenberg form, Numer. Math. 12 (1968), no. 5, 349-368; Available online at http://dx.doi.org/10.1007/BF02161358

[8] F. Qi, A determinantal representation for derangement numbers, Glob. J. Math. Anal. 4 (2016), no. 3, 17-17; Available online at http://dx.doi.org/10.14419/gjma.v4i3.6574

[9] F. Qi, Derivatives of tangent function and tangent numbers, Appl. Math. Comput. 268 (2015), 844-858; Available online at http://dx.doi.org/10.1016/j.amc.2015.06.123

[10] F. Qi and R. J. Chapman, Two closed forms for the Bernoulli polynomials, J. Number Theory 159 (2016), 89-100; Available online at http://dx.doi.org/10.1016/j.jnt.2015.07.021

[11] F. Qi and B.-N. Guo, Explicit formulas for derangement numbers and their generating function, J. Nonlinear Funct. Anal. 2016 (2016), Article ID 45, 9 pages.

[12] F. Qi and B.-N. Guo, Expressing the generalized Fibonacci polynomials in terms of a tridiagonal determinant, Matematiche (Catania) 71 (2016), no. 2, in press.

[13] F. Qi, X.-T. Shi, and F.-F. Liu, Several identities involving the falling and rising factorials and the Cauchy, Lah, and Stirling numbers, ResearchGate Research (2015), available online at http://dx.doi.org/10.13140/RG.2.1.2462.1606

[14] F. Qi, J.-L. Wang, and B.-N. Guo, A recovery of two determinantal representations for derangement numbers, Cogent Math. (2016), 3: 1232878, 7 pages; Available online at http: //dx.doi.org/10.1080/23311835.2016.1232878

[15] F. Qi, J.-L. Wang, and B.-N. Guo, A representation for derangement numbers in terms of a tridiagonal determinant, Kragujevac J. Math. (2017), in press.

[16] C.-F. Wei and F. Qi, Several closed expressions for the Euler numbers, J. Inequal. Appl. 2015, 2015:219, 8 pages; Available online at http://dx.doi.org/10.1186/s13660-015-0738-9.

[17] H. S. Wilf, generatingfunctionology, Second edition, Academic Press, Inc., Boston, MA, 1994.

[18] H. S. Wilf, generatingfunctionology, Third edition. A K Peters, Ltd., Wellesley, MA, 2006.

$U R L:$ https://qifeng618.wordpress.com

$U R L:$ http://www.researchgate.net/profile/Bai-Ni_Guo/ 